\title{
Expression of $L R P$ Gene in Breast Cancer Patients Correlated with MRP1 as Two Independent Predictive Biomarkers in Breast Cancer
}

\author{
Mohsen Taheri ${ }^{1,2}$, Jamshid Motalebzadeh ${ }^{1}$, Frouzandeh Mahjoubi $^{1 *}$
}

\begin{abstract}
Background: Breast cancer is the most common malignancy in women. Multidrug resistance (MDR) is still a great obstacle of breast cancer chemotherapy. We have previously shown that multidrug resistance-associated protein 1 (MRPI) is associated with response to neoadjuvant chemotherapy. The lung resistance-related protein $(L R P)$ is identified as a prognostic marker and response to treatment factor which has been studied mainly in hematological malignancy and leukemia. In this study, we aimed to analyze $L R P$ expression and possible correlation between the expression level of this gene with $M R P 1$ as a candidate marker for chemotherapy resistance. Materials and Methods: We collected 54 breast tumors and adjacent normal tissues from Iranian breast cancer patients and Real time RT-PCR was employed to measure the gene expression level in our samples. Results: MRPl and LRP expression level were significantly lower in tumor tissues of the patients responding to chemotherapy compared to non-responding patients. No relation between the expression level of either of these genes and clinicopathology markers was found. Conclusion: Our results suggest that $L R P$ gene expression is correlated to $M R P 1$ in human breast cancer cells and may affect the clinical response to treatment.
\end{abstract}

Keywords: Multidrug resistance- Breast cancer- LRP- MVP- MRP1

Asian Pac J Cancer Prev, 19 (11), 3111-3115

\section{Introduction}

Breast cancer with an estimated 1.7 million cases and 521,900 deaths in 2012 is known as the most common malignancy in women. Breast cancer alone accounts for $25 \%$ of all cancer cases and $15 \%$ of all cancer deaths among females. In developed countries, breast cancer consists about $38 \%$ of deaths (Torre et al., 2015) and in Iran with $23 \%$ frequency in women is the most prevalent cancer in women (Mousavi et al., 2008).

Among solid tumors, breast cancer is considered as one of the most responsive to chemotherapy, but most of the tumors that initially response to drugs become resistance (Burger et al., 2003) and the incidence of resistance increases with breast cancer progression (Martin et al., 2014). This phenomenon is named as Multi Drug Resistance (MDR) in which cancer cells become resistant to the wide spectrum of drugs with different structure and function (Gottesman, 2002). Several mechanisms responsible for drug resistance have been identified including mutation in or overexpression of the drug's target, drug inactivation and efflux of the drug from the cell by ATP Binding Cassette (ABC) transporters
(Motalebzadeh et al., 2017). These transporters are capable of decreasing the intracellular drug concentration in vitro (Dean, 2009).

The Multi Drug Resistance Related Protein1 (MRP1) is a member of the ABC-transporter that located on chromosome $16 \mathrm{p} 13$ and transports a wide range of compounds including glutathione conjugates and cyclic nucleotides out of cells. It has been shown MRPI molecule has a role in the efficiency of the drugs used for the treatment of nonmalignant diseases such as antibiotics and antiviral drugs (Cole, 2014).

Another mechanism of drug resistance is the intracellular redistribution of drugs without changing their intracellular accumulation (Lee et al., 2017). Lung Resistance Protein $(L R P)$ is not a member of $\mathrm{ABC}$ transporter family but it is a major vault protein (MVP) and is found in cytoplasm and nuclear membrane and can transport drugs out of nucleus. In cells, LRP is usually associated with vesicles and lysosomes and is thought to be responsible for the uptake of drugs in cytoplasmic vesicles that are then probably extruded from the cell by exocytose (Triller et al., 2006). LRP gene is located on chromosome $16 \mathrm{p} 11.2$ close to the MRPl gene. Because of 
its position on nuclear membrane it is thought to have an effect on the transport of drugs that their main target is DNA therefore it plays an important role in drug resistance (Lu and Shervington, 2008).

The role of MRP1 in inducing drug resistance in many cancers has been widely investigated. In our previous study we found that MRPl is correlated with response to the chemotherapy in Iranian breast cancer patients (Taheri and Mahjoubi, 2013). The effect of $L R P$ in prognosis and response to treatment has been studied mainly in hematological malignancy and leukemia and rarely on solid tumors (Bhatia et al., 2015).

The aim of the present study was to investigate the role of $L R P$ and $M R P 1$ genes in response to chemotherapy and the prognostic value of these genes in Iranian breast cancer patients.

\section{Materials and Methods}

\section{Patients sample collection}

Fifty four patients with breast cancer who had undergone breast cancer surgery were enrolled in this study. The project was approved by the local ethical committee of National Institute of Genetic Engineering and Biotechnology (NIGEB). Written informed consent was obtained from all cases. Tissue specimens (tumor and normal tissue adjacent to tumor) were collected by the surgeons. Histologic diagnosis was confirmed for all samples. The patients' features are shown in Table 1. The patients then were received anthracycline based chemotherapy (FAC/FEC). Response to treatment was evaluated by UICC criteria. Routine clinical examination comprises complete blood examination, chest radiography, ECG (Echocardiography), bone scan and liver function tests were done.

\section{RNA extraction and cDNA synthesis}

RNA extraction was carried out by Tripure Isolation Reagent (Roch applied sciences). For cDNA synthesis, $1 \mu \mathrm{g}$ of total RNA from each sample was used to synthesize first-strand cDNA according to the manufacturer's protocol (Frementas).

\section{Real-time qRT-PCR}

The mRNA levels of $L R P$ gene was measured by real-time qRT-PCR using a lightcycler ${ }^{\mathrm{TM}}$ system (Roche Applied Sciences) with Fast-Start DNA Master SYBR-Green I kit (Roche Applied Sciences). In addition, the mRNA level of $\beta$-actin as internal control was measured and used to normalize the mRNA levels of the drug resistance gene.

The primers were designed using Primer Premier 5.0 software (Table 2).

All reactions were carried out in a total volume of $20 \mu \mathrm{L}$ in capillary tubes. Each reaction mix contained $0.6 \mu \mathrm{M}$ of each primer, $2.5 \mathrm{mM} \mathrm{MgCl}_{2}$ and $2 \mu \mathrm{L}$ of Fast Start Master solution. A total of $18 \mu \mathrm{L}$ of this reaction mix was placed into glass capillaries, and $2 \mu \mathrm{L}$ of cDNA was added as template. The capillary tubes were capped and placed in the carousel under reduced light conditions. Thermal cycling consisted of an initial denaturation step $95^{\circ} \mathrm{C}$ for 10 min followed by an amplification program (denaturation, amplification and extension) repeated for 55 cycles with temperature ramp rate of $20{ }^{\circ} \mathrm{C} / \mathrm{sec}$. The amplification program was $95^{\circ} \mathrm{C}$ for $10 \mathrm{sec}$, primer Tm (Table 2) for $15 \mathrm{sec}$ and $72^{\circ} \mathrm{C}$ for $15 \mathrm{sec}$ with a single fluorescence acquisition at the end of the elongation step. The third segment consisted of a melting curve program at $95^{\circ} \mathrm{C}$ for $0 \mathrm{sec}, 70^{\circ} \mathrm{C}$ for $10 \mathrm{sec}$ and $95^{\circ} \mathrm{C}$ for $0 \mathrm{sec}$ with a linear temperature transition rate of $0.1^{\circ} \mathrm{C} / \mathrm{sec}$ with continuous fluorescence acquisition. Finally, a cooling program cooled the reaction mixture to $40^{\circ} \mathrm{C}$.

For confidence accuracy and specificity of Real Time PCR, PCR products were checked on 1.5\% agarose gel.

A standard Lightcycler PCR program was established using logarithmic regression for each gene.

\section{Data Analysis}

The raw data were analyzed using version 3.03 of the Lightcycler software as described previously (Golalipour et al., 2007). Statistical analysis was performed using SPSS for software V16.0 (SPSS, Inc., Chicago, IL). Differences between groups were analyzed by one-way analysis of variance (ANOVA) and the Tuckey multiple comparison tests. Association between clinical characteristics and an expression level was determined using Chi-squared test. A p-value less than 0.05 were considered statistically significant. Also to determine the relationship between the expression level of $L R P$ and $M R P 1$ gene the spearman correlation coefficient was used.

\section{Results}

This study was done on 54 breast cancer patients to assess the expression levels of MRP1 and LRP on clinicopathology criteria and response to treatment.

$L R P$ gene expression level in tumor and normal breast tissues was assessed by Real Time RT PCR. The final results were expressed as the ratio of each gene to $\beta$-Actin, an internal control gene, in each sample as described previously (Motalebzadeh et al., 2018).

Expression of LRP in normal and tumoral breast tissues and its correlation with MRPI

According to our previous study, we found the expression level of $M R P 1$ in normal and tumor tissues

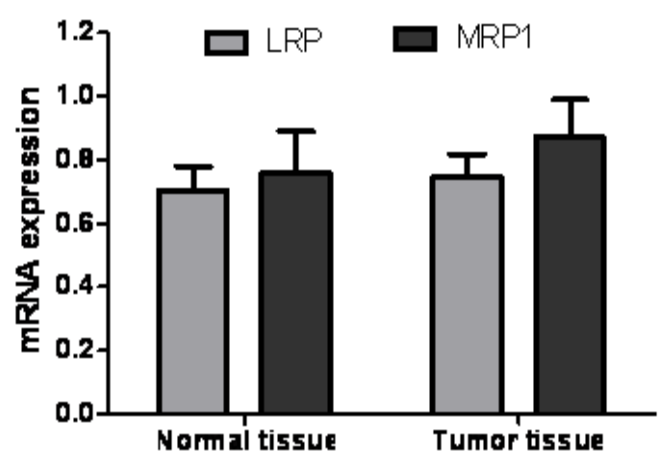

Figure 1. Relative Mean Expression of MRP1 and LRP in Normal and Tumor Breast Tissue 
Table 1. Patients and Tumor Characteristics

\begin{tabular}{lc}
\hline Characteristic & No (\%) \\
\hline All of the Patients & $54(100 \%)$ \\
Menopausal Status & \\
Premenopausal & $22(41 \%)$ \\
Postmenopausal & $32(59 \%)$ \\
Pathological Type & \\
Ductal carcinoma & $48(89 \%)$ \\
Lobular carcinoma & $6(11 \%)$ \\
Histological Grade & \\
Grade-I & $9(17 \%)$ \\
Grade-II & $30(55 \%)$ \\
Grade-III & $15(28 \%)$ \\
Tumor Size & \\
$>5 \mathrm{~cm}$ & $27(50 \%)$ \\
$5-8 \mathrm{~cm}$ & $13(24 \%)$ \\
$8-10 \mathrm{~cm}$ & $6(11 \%)$ \\
$>10 \mathrm{~cm}$ & $8(15 \%)$ \\
Lymph Node Metastasis & \\
Positive & $34(63 \%)$ \\
Negative & $20(37 \%)$ \\
Response to Chemotherapy & \\
Responder & $40(74 \%)$ \\
Non-responder & $14(26 \%)$ \\
\hline
\end{tabular}

were $0.7560 \pm 0.13348$ and $0.8709 \pm 0.11724$, respectively (Taheri and Mahjoubi, 2013).

As shown in Figure 1, the LRP mRNA level of breast tumoral tissues $(0.7458 \pm 0.07198)$ was significantly $(\mathrm{p}=0.003)$ higher than normal tissue $(0.7029 \pm 0.07544)$.

The results showed that there was a positive significant correlation between expression levels of MRP1 and LPR in breast tumor tissues $(\mathrm{r}=0.6420, \mathrm{P}<0.0001)$ and normal breast tissues ( $\mathrm{r}=0.4937, \mathrm{P}=0.0002)$ (Figure 2).

Response to chemotherapy and correlation with LRP gene expression level

The clinical response showed that 40 (74\%) patients were classified as responsive (R) and $14(26 \%)$ as nonresponsive (NR). Relative expression of $L R P$ gene was $0.7264 \pm 0.07035$ and $0.8012 \pm 0.04244$, respectively (Figure 3). The results showed that the expression level of $L R P$ was significantly higher in nonresponder than responder patients $(\mathrm{P}<0.05)$.

Clinicopathology characteristics and LRP gene expression level

We investigated the mRNA expression level of $L R P$
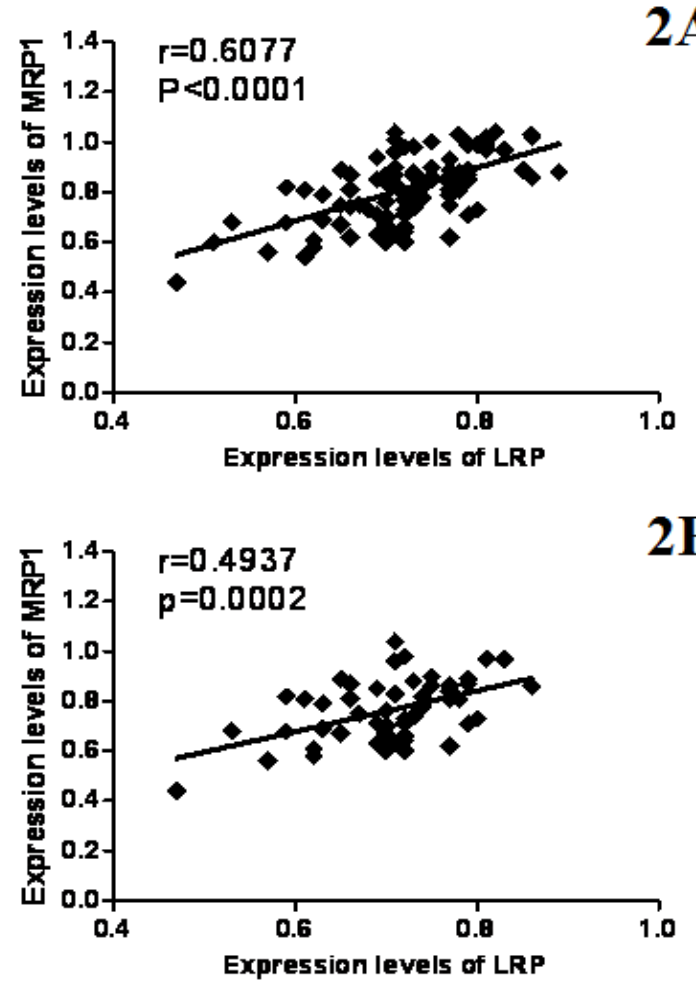

Figure 2. Correlation between the Expression Levels of $M R P 1$ and $L P R$ in Breast Tumor Tissue (2A) and Normal Breast Tissue (2B).

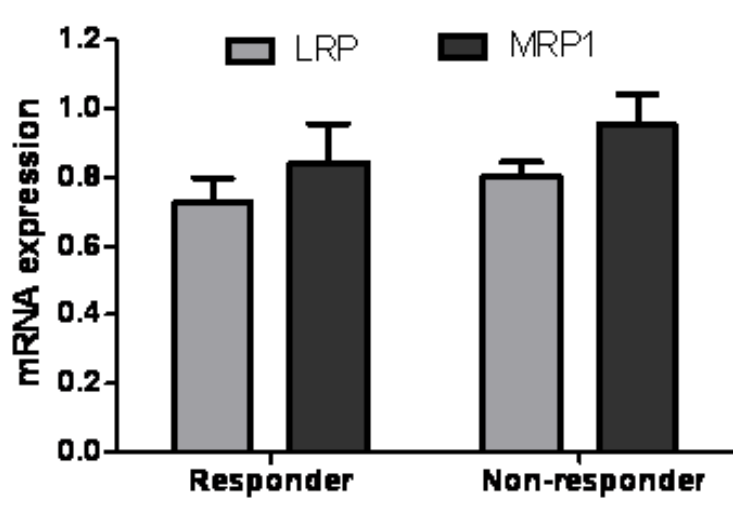

Figure 3. Relative Mean Expression of MRP1 and LRP in Responder and Non-responder

gene in relation to traditional clinical prognostic factors as listed in Table 2. The average age of the patients was calculated $47.26(\mathrm{SD}=13.89)$ years. Of 54 patients, 9 $(17 \%)$ were in grade-I, $30(55 \%)$ were in grade-II and $15(28 \%)$ were in grade-III. There was no association between the expression of $L R P$ and grade of breast tumor. Concerning to tumor size, 27 (50\%) patients had tumor

Table 2. Sequences of the Primers

\begin{tabular}{lclc}
\hline Primer & Amplicon size & \multicolumn{1}{c}{ Sequence } & $\mathrm{T}_{\mathrm{m}}$ \\
\hline LRP-Forward & $137 \mathrm{bp}$ & 5' GGC AGG ACA ATG AGA GGG TAC T 3' & $60^{\circ}$ \\
LRP-Reversed & & 5' CCG AAC TTG CCC TGT GAC AT 3' & \\
$\beta$-actin-Forward & $161 \mathrm{bp}$ & 5'-GAG ACC TTC AAC ACC CCA GCC-3' & $60^{\circ}$ \\
$\beta$-actin-Reversed & & 5'-AGA CGC AGG ATG GCA TGG G-3' & \\
\hline
\end{tabular}


size $<5 \mathrm{~cm}, 13$ patients $(24 \%)$ had tumor size between $5-8 \mathrm{~cm}, 6(11 \%)$ between $8-10 \mathrm{~cm}$ and $8(15 \%)$ over 10 $\mathrm{cm}$. Our results indicated there was not any association between tumor size and the expression level of $L R P$. Sixty-nine percent of patients were estrogen receptor positive and $62 \%$ were progesterone receptor positive. In $63 \%$ of patient's lymph node was involved. There was no association between hormone status, lymph node involvement and expression level.

\section{Discussion}

Although breast cancer survival rates have increased in recent years many women still die from the disease. Resistance to chemotherapy with metastasis is the most important cause of death (Fonseca, 2012). Resistance to chemotherapy limits the effectiveness of anti neoplastic treatment. Several proteins have been identified that are able to prevent the intracellular accumulation of anticancer agents by efflux mechanism such as MDR1 and MRP1 (Fojo and Coley, 2007). Several studies showed that $L R P$ promotes cancer progression and the expression of this gene is an adverse prognostic factor for response to chemotherapy (Huh et al., 2006; Lee et al., 2017). The majority of the studies have been performed in hematological malignancies. Only a few studies have addressed the role of $L R P$ expression in solid tumors and no clear relationship between $L R P$ and outcome of the patients was observed (Gillet and Gottesman, 2010; Martin et al., 2014).

In the present study, we examined the expression level of $L R P$ gene and the correlation of this gene with $M R P 1$ in breast cancer patients. The $L R P$ and $M R P 1$ were studied by real time RT PCR and their clinical significance in chemoresistance were also investigated.

With respect to $L R P$, we found that a statistically significant correlation between the expression level and response to treatment in this group of patients. Herman Burger et al in a study done on breast cancer patients found a statistically significant correlation between the expression level and length of PFS (progression-free survival) (Burger et al., 2003). In another study by Schneider and coworkers, they found $L R P$ expression in locally advanced breast cancer was associated with the presence of axillary nodal metastasis after induction chemotherapy, and they suggested that $L R P$ may play a role in the resistance phenotype of intrinsically resistant clones that persist after induction chemotherapy (Schneider et al., 2001). The fact that $\mathrm{ABC}$ transporter expression levels and resistance to chemotherapy are positively correlated in some but not all breast cancer may reflect differences in analytic methods, patients' population, or the chemotherapeutic drugs used.

Overexpression of MRP1 has been observed in several human cancers, including breast cancer, gastric hepatoid adenocarcinoma, and several types of leukemia (Faneyte et al., 2004; Mahjoubi et al., 2008; Iseri et al., 2011). We previously found and reported a significant positive correlation between $M R P 1$ expression and response to treatment and the importance of $M R P 1$ rather than MDR1 in breast cancer patients (Taheri and Mahjoubi, 2013).
Atalay and co-workers observed increased expression of MRP1 after chemotherapy in almost all locally advanced breast cancer patients but this increase was not statistically significant (Atalay et al., 2006). Rudas et al demonstrated that the expression of $M R P 1, L R P$ and $\mathrm{p}$-gp increase after preoperative chemotherapy but there was no association between their expression and response to chemotherapy (Rudas et al., 2003). A correlation between $M R P 1$ expression and patient survival rates after chemotherapy has been noted in some studies (Burger et al., 2003; Rudas et al., 2003), whereas other reports showed no correlation between $M R P 1$ expression and prognosis (Faneyte et al., 2004; Huh et al., 2006).

We also found that the expression level of $L R P$ was positively related with $M R P 1$ gene and this may indicate that the expression of these genes is likely to be coordinately regulated. Similar correlations between the expression of these genes were observed previously (Burger et al., 2003)

Regarding to relationship between the expression and clinicopathology markers, no association was found between expression of these genes and stage of tumor. There was also no association between tumor size, hormone status, lymph node involvement and expression level of $L R P$ and MRP1 genes.

In conclusion the results showed that MRP1/LRP expression has been associated with unfavorable outcome in our breast cancer patients. Both $L R P$ and $M R P 1$ seem to contribute to drug resistance. Determination of $M R P 1$ and $L R P$ (either alone or in combination) may be important for the prediction of the chemotherapy outcome in breast cancer patients. It may also be valuable for developing anticancer drugs with more effectiveness on the patients.

Further studies should focus on the molecular basis of how the expression of these transporters is regulated in normal breast cells and in their malignant counterparts.

\section{Compliance with Ethical Standards}

Informed consent was obtained from all individual participants included in the study.

\section{Conflict of Interest}

All authors declare that they have no conflict of interest.

\section{Acknowledgements}

This research was financially supported by the National Institute of Genetic Engineering and Biotechnology, Tehran, Iran.

\section{References}

Atalay C, Gurhan ID, Irkkan C, et al (2006). Multidrug resistance in locally advanced breast cancer. Tumor Biol, 27, 309-18.

Bhatia P, Masih S, Varma N, et al (2015). High Expression of Lung Resistance Protein mRNA at diagnosis predicts poor early response to induction chemotherapy in childhood acute lymphoblastic leukemia. Asian Pac J Cancer Prev, 16, 6663-8.

Burger H, Foekens JA, Look MP, et al (2003). RNA expression of breast cancer resistance protein, lung resistance-related 
protein, multidrug resistance-associated proteins 1 and 2, and multidrug resistance gene 1 in breast cancer. Clin Cancer Res, 9, 827-36.

Cole SP (2014). Multidrug resistance protein 1 (MRP1, ABCC1): A'Multitasking'ABC transporter. J Biol Chem, 114, 609248.

Dean M (2009). ABC transporters, drug resistance, and cancer stem cells. J Mammary Gland Biol Neoplasia, 14, 3-9.

Faneyte IF, Kristel PM, Van De Vijver MJ (2004). Multidrug resistance associated genes MRP1, MRP2 and MRP3 in primary and anthracycline exposed breast cancer. Anticancer Res, 24, 2931-40.

Fojo T, Coley HM (2007). The role of efflux pumps in drug-resistant metastatic breast cancer: new insights and treatment strategies. Clin Breast Cancer, 7, 749-56.

Gillet JP, Gottesman MM (2010). Mechanisms of multidrug resistance in cancer. Methods Mol Biol, 596, 47-76.

Golalipour M, Mahjoubi F, Sanati MH (2007). Gene dosage is not responsible for the upregulation of $M R P 1$ gene expression in adult leukemia patients. Arch Med Res, 38, 297-304.

Gottesman MM (2002). Mechanisms of cancer drug resistance. Ann Revi Med, 53, 615-27.

Huh HJ, Park CJ, Jang S, et al (2006). Prognostic significance of multidrug resistance gene 1 (MDR1), multidrug resistance-related protein (MRP) and lung resistance protein $(L R P)$ mRNA expression in acute leukemia. $J$ Korean Med Sci, 21, 253-8.

Iseri OD, Mutlu P, Ferit A, et al (2011). Expression of multidrug resistance 1 , lung resistance protein and breast cancer resistance protein genes in chronic leukemias. Int $J$ Hematol Oncol, 27, 92-100.

Lee HM, Joh JW, Seo SR, et al (2017). Cell-surface major vault protein promotes cancer progression through harboring mesenchymal and intermediate circulating tumor cells in hepatocellular carcinomas. Sci Rep, 7, 13201.

$\mathrm{Lu}$ C, Shervington A (2008). Chemoresistance in gliomas. Mol Cell Biochem, 312, 71-80.

Mahjoubi F, Golalipour M, Ghavamzadeh A, et al (2008). Expression of MRP1 gene in acute leukemia. Sao Paulo Med $J, \mathbf{1 2 6}, 172-9$.

Martin HL, Smith L, Tomlinson DC (2014). Multidrug-resistant breast cancer: current perspectives. Breast Cancer, 6, 1 .

Motalebzadeh J, Mahjoubi F, Nafissi N, et al (2017). FBLN-4 and BCRP genes as two prognostic markers are downregulated in breast cancer tissue. Cancer Biomarkers, 19, 51-5.

Motalebzadeh J, Shabani S, Rezayati S, et al (2018). Prognostic value of FBXO39 and ETS-1 but not BMI-1 in Iranian colorectal cancer patients. Asian Pac J Cancer Prev, 19, 1357-62.

Mousavi SM, Gouya MM, Ramazani R, et al (2008). Cancer incidence and mortality in Iran. Ann Oncol, 20, 556-63.

Rudas M, Filipits M, Taucher S, et al (2003). Expression of $M R P 1, L R P$ and Pgp in breast carcinoma patients treated with preoperative chemotherapy. Breast Cancer Res Treat, 81, 149-57.

Schneider J, Gonzalez-Roces S, Pollán M, et al (2001). Expression of $L R P$ and MDR1 in locally advanced breast cancer predicts axillary node invasion at the time of rescue mastectomy after induction chemotherapy. Breast Cancer Res, 3, 183.

Taheri M, Mahjoubi F (2013). MRP1 but not MDR1 is associated with response to neoadjuvant chemotherapy in breast cancer patients. Dis Markers, 34, 387-93.

Torre LA, Bray F, Siegel RL, et al (2015). Global cancer statistics, 2012. CA Cancer J Clin, 65, 87-108.

Triller N, Korošec P, Kern I, et al (2006). Multidrug resistance in small cell lung cancer: expression of P-glycoprotein, multidrug resistance protein 1 and lung resistance protein in chemo-naive patients and in relapsed disease. Lung Cancer, $\mathbf{5 4}, 235-40$.

\section{cc)}

This work is licensed under a Creative Commons AttributionNon Commercial 4.0 International License. 